\title{
Bericht aus dem ÖGPP-Vorstand
}

\author{
Christoph Stuppäck
}

\section{Liebe Kolleginnen und Kollegen,}

Trotz Beginns ihrer Gültigkeit mit Februar 2007 ist die neue Ausbildungsordnung immer noch nicht umgesetzt ich habe an dieser Stelle bereits einige Male darüber berichtet. Nach wie vor sieht es so aus, als arbeiteten einige Kräfte am Kippen dieser neuen Ausbildungsordnung für Psychiatrie und Psychotherapeutische Medizin zugunsten der alten Facharztregelung. Es steht jedoch zu hoffen, dass demnächst in der Österreichischen Ärztekammer und dem Ministerium entscheidende Weichen gestellt werden. Erfreulich ist, dass die 3 öffentlichen Universitätskliniken für Psychiatrie und Psychotherapie (Wien, Graz, Innsbruck) sowie die private Universitätsklinik für Psychiatrie und Psychotherapie I der PMU in Salzburg von den lokalen Ausbildungskommissionen die Ausbildungsbefugnis erhalten haben.

Mehrere neue Ärztekammerdiplome sind in Entwicklung, vom Vorstand der ÖGPP werden einerseits eines über Sexualmedizin, andererseits eines über Schlafmedizin forciert, beide Diplome zeichnen sich durch eine erfreuliche Interdisziplinarität der Curricula aus.

Eine Novellierung des Unterbringungsgesetzes schreitet ebenfalls voran, eine Verzögerung ergab sich zuletzt durch die Haltung der Österreichischen Gesellschaft für Kinder- und Jugendpsy-

Univ.-Prof. Dr. Christoph Stuppäck Universitätsklinik für Psychiatrie und Psychotherapie I, Christian-Doppler-Klinik, Ignaz-Harrer-Straße 79, 5020 Salzburg, Österreich, E-Mail: c.stuppaeck@salk.at chiatrie: Die Proponenten dieser Gesellschaft halten einen Verzicht auf ein zweites Zeugnis für indiskutabel. Unterschiedliche gesetzliche Regelungen für Kinder/Jugendliche bzw. Erwachsene scheinen wahrscheinlich und sind auch gut argumentierbar, es ist also zu hoffen, dass demnächst die Innovationen, über die bereits hier berichtet wurde, in Kraft treten, dadurch klare administrative Erleichterungen bei gleichbleibender Rechtssicherheit für unsere Patientinnen und Patienten erreicht werden können.

Berichte aus dem Vorstand haben natürlich auch darauf einzugehen, dass der Vorstand seit der Generalversammlung anlässlich der letzten Jahrestagung im April in Gmunden eine andere $\mathrm{Zu}-$ sammensetzung hat: Statutengemäß sind Margit Wrobel und Angelika Rießland-Seifert aus ihren Funktionen als Schatzmeisterin respektive Sekretärin der ÖGPP ausgeschieden. Beide haben in den vier Jahren ihrer Zugehörigkeit zum Vorstand mit hohem Engagement und hoher fachlicher Kompetenz sehr viel für die Österreichische Psychiatrie geleistet, beide werden auch weiterhin der ÖGPP „zuarbeiten“: so wird Angelika Rießland-Seifert für die weitere Planung und Entwicklung im CL-Bereich mit dem ÖBIG in Verbindung, Margit Wrobel wird Vorsitzende der Prüfungskommission bleiben.

Sie haben ihren Nachfolgerinnen, Dr. Christa Rados (Schatzmeisterin) und PD Dr. Claudia Klier (Sekretärin) die Latte hoch gelegt. Diese beiden wurden von Nominierungskomitee und Vorstand als einzige Kandidatinnen genannt und in der Generalversammlung jeweils einstimmig in diese Funktionen gewählt. Die ersten offiziellen Auftritte im neuen Amt fanden am 15. Juni 2009 bei einer Vorstandssitzung statt, demnächst wird auch wieder das Programmkomitee für die Jahrestagung 2010 (22. bis 24. April in Gmunden) zusammentreten.

Gmunden 2009 war in vielerlei Hinsicht der bisher erfolgreichste Kongress seit Bestehen der ÖGPP: über 750 Teilnehmer, dazu mehr als 200 Vertreter der Industrie und - das ist besonders erfreulich - 412 Teilnehmer am Schülerkongress trugen zu einem „Rekordergebnis" bei. Die Gesamtbeurteilung des Kongresses durch seine Besucher ergab einen Schnitt von 1.3, was die hohe Qualität dieser Veranstaltung widerspiegelt.

Wie immer möchte ich abschließen, indem ich Sie ersuche, die Arbeit des Vorstands der ÖGPP sowie aller Leiterinnen und Leiter von Arbeitsgruppen und Sektionen nicht nur wohlwollend oder kritisch zur Kenntnis zu nehmen, sondern sich auch persönlich so stark wie möglich zu engagieren. Mag der Termin der letzten Generalversammlung ganz am Ende des Kongresses nicht allzu günstig gelegen sein, so war trotzdem der schüttere Besuch einziger Wermutstropfen dieser herausragenden Veranstaltung. Wenden Sie sich also mit Engagement, Anregungen oder Kritik jederzeit an das Sekretariat der Gesellschaft (E-mail: sylvia.blebann@aon.at ) oder direkt an eines der Vorstandsmitglieder.

Univ.-Prof. Dr. Christoph Stuppäck Past Präsident der ÖGPP 